\title{
NEW TRIGONOMETRIC AND HYPERBOLIC INEQUALITIES
}

\author{
BARKAT ALI BHAYO, RIKU KLÉN, AND JÓZSEF SÁNDOR
}

Received 24 February, 2015

\begin{abstract}
The aim of this paper is to prove new trigonometric and hyperbolic inequalities, which constitute refinements or analogs of famous Cusa-Huygens, Wu-Srivastava, and related inequalities. Most of the results are sharp.
\end{abstract}

2010 Mathematics Subject Classification: 26D05; 26D07; 33B10

Keywords: circular functions, hyperbolic functions, trigonometric inequalities, hyperbolic inequalities

\section{INTRODUCTION}

Since the last decade many authors have been interested in finding upper and lower bounds for expression $f(x) / x$, where $f(x)$ is a trigonometric or a hyperbolic function. We continue this line of research.

The inequalities

$$
(\cos x)^{1 / 3}<\frac{\sin x}{x}<\frac{\cos x+2}{3}
$$

hold for $0<|x|<\pi / 2$. The left hand side inequality is due to by D.D. Adamović and D.S. Mitrinović [9, p. 238], and the right hand side one was obtained by N. Cusa and C. Huygens in 2005 [16]. The hyperbolic version of (1.1) is

$$
(\cosh x)^{1 / 3}<\frac{\sinh x}{x}<\frac{\cosh x+2}{3},
$$

for $x \neq 0$. The left hand side inequality in (1.2) was obtained by Lazarević $[9$, p. 270], and the right hand side inequality is called the hyperbolic Cusa-Huygens inequality [11].

In 1989 J. Wilker [10] discovered the following inequality

$$
\left(\frac{\sin x}{x}\right)^{2}+\frac{\tan x}{x}>2, \quad 0<|x|<\frac{\pi}{2} .
$$

The third author has been supported by a Grant GSC-30249-2015 of Babes-Bolyai University of Cluj, Romania. 
Numerous authors have studied this inequality by giving simpler proofs and generalization, e.g, see $[2,3,11,12,17-19,21,22]$. The following inequality is due to Huygens [5]

$$
2 \frac{\sin x}{x}+\frac{\tan x}{x}>3, \quad 0<|x|<\frac{\pi}{2}
$$

and is called Huygens inequality or the first Wilker inequality. In [18], Wu and Srivastava introduced the following inequality

$$
\left(\frac{x}{\sin x}\right)^{2}+\frac{x}{\tan x}>2, \quad 0<|x|<\frac{\pi}{2},
$$

which is called the second Wilker inequality.

We study inequalities (1.1)-(1.5) and our main results are the following five theorems. Our first main result is a generalization of Cusa-Huygens inequality (1.1).

Theorem 1. For $x \in[-\pi / 2, \pi / 2]$, we have

$$
\frac{\cos x+\alpha-1}{\alpha} \leq \frac{\sin x}{x} \leq \frac{\cos x+\beta-1}{\beta},
$$

with the best possible constants $\alpha=\pi /(\pi-2) \approx 2.75194$ and $\beta=3$. The lower bound is sharp for $x \in\{-\pi / 2,0, \pi / 2\}$ and the upper bound is sharp for $x=0$.

Remark 1. The upper bound of Theorem 1 is sharp at point $x=0$ and the lower bound at points $x=-\pi / 2, x=0$ and $x=\pi / 2$. For values $x \in[-\pi / 2, \pi / 2]$ the difference between the function and the lower bound is less than 0.01 and the difference between the function and the upper bound is less than 0.031 . The right hand side inequality holds true for all real numbers $x$ and as a sharp inequality for all $x \neq 0$.

In the following two theorems we introduce Wu-Srivastava type inequalities for the trigonometric functions.

Theorem 2. For $x \in[-\pi / 2, \pi / 2]$, we have

$$
\left(\frac{x}{\sin x}\right)^{2}+\left(\frac{\pi^{2}}{4}-1\right) \frac{x}{\tan x} \leq \frac{\pi^{2}}{4}
$$

and the equality is attained for $x=-\pi / 2, x=0$ and $x=\pi / 2$.

Remark 2. The upper bound of Theorem 2 is sharp at points $x=-\pi / 2, x=0$ and $x=\pi / 2$. For values $x \in[-\pi / 2, \pi / 2]$ the difference between the function and the upper bound is less than 0.13 .

Theorem 3. For $x \in[-\pi / 2, \pi / 2]$, we have

$$
\begin{aligned}
(\alpha-1) \frac{x}{\sin x}+\frac{x}{\tan x} & \leq \alpha, \\
\left(\frac{x}{\sin x}\right)^{\alpha}+\frac{x}{\tan x} & <\left(\frac{\pi}{2}\right)^{\alpha},
\end{aligned}
$$

with the best possible constant $\alpha=\pi /(\pi-2)$. The inequality (1.8) holds as equality for $x \in\{-\pi / 2,0, \pi / 2\}$ and the inequality (1.9) holds as equality for $x \in\{-\pi / 2, \pi / 2\}$. 
Remark 3. For values $x \in[-\pi / 2, \pi / 2]$ the difference between the function $\alpha$ and the lower bound is less than 0.031. In inequality (1.9) the difference between the function and the lower bound is between 1.45 and 1.9.

The next theorem is a generalization of the Huygens inequality.

Theorem 4. For $x \in(-\pi / 2, \pi / 2)$, we have

$$
3 \cos x \leq \frac{x}{\sin x}+2 \frac{x}{\tan x} \leq 2+\cos x .
$$

The inequalities hold as equalities for $x=0$.

The following result is a Wilker and Wu-Srivastava type result for the hyperbolic functions.

Theorem 5. For $x>0$, we have

$$
\left(\frac{x}{\sinh x}\right)^{2}+\frac{x}{\tanh x}<\left(\frac{\sinh x}{x}\right)^{2}+\frac{\tanh x}{x}<\frac{1+\cosh (2 x / 3)}{2}\left(\left(\frac{x}{\sinh x}\right)^{2}+\frac{x}{\tanh x}\right) \text {. }
$$

Our last result is a counterpart of the inequality

$$
\exp \left(-x^{2} / 6\right)<\frac{2+\cos x}{3},
$$

$x \in(0, \infty)$, which recently appeared in [20, Thm 2].

Theorem 6. For $x \in(0, \pi / 2)$, the following inequalities hold

$$
\exp \left(\alpha-\frac{(\pi-2) x^{2}}{2 \pi}\right)<\frac{(\pi-2) \cos (x)+2}{\pi}<\exp \left(\beta-\frac{(\pi-2) x^{2}}{2 \pi}\right),
$$

with the best possible constants $\alpha=\left(\pi^{2}+8 \log (2 / \pi)-2 \pi\right) / 8 \approx-0.00328$ and $\beta=0$.

\section{PReliminaries AND Lemmas}

The following lemma was originally proved in [13], but can also be found in [4].

Lemma 1. For $0<R \leq \infty$. Let $A(x)=\sum_{n=0}^{\infty} a_{n} x^{n}$ and $C(x)=\sum_{n=0}^{\infty} c_{n} x^{n}$ be two real power series converging on the interval $(-R, R)$. If the sequence $\left\{a_{n} / c_{n}\right\}$ is increasing (decreasing) and $c_{n}>0$ for all $n$, then the function $A(x) / C(x)$ is also increasing (decreasing) on $(0, R)$.

For $|x|<\pi$, the following power series expansions can be found in [7, 1.3.1.4 (2)-(3)],

$$
x \cot x=1-\sum_{n=1}^{\infty} \frac{2^{2 n}}{(2 n) !}\left|B_{2 n}\right| x^{2 n},
$$




$$
\cot x=\frac{1}{x}-\sum_{n=1}^{\infty} \frac{2^{2 n}}{(2 n) !}\left|B_{2 n}\right| x^{2 n-1}
$$

and

$$
\operatorname{coth} x=\frac{1}{x}+\sum_{n=1}^{\infty} \frac{2^{2 n}}{(2 n) !}\left|B_{2 n}\right| x^{2 n-1},
$$

where $B_{2 n}$ are the even-indexed Bernoulli numbers, see [6, p. 231]. We can get the following expansions directly from (2.2) and (2.3),

$$
\begin{aligned}
& \frac{1}{(\sin x)^{2}}=-(\cot x)^{\prime}=\frac{1}{x^{2}}+\sum_{n=1}^{\infty} \frac{2^{2 n}}{(2 n) !}\left|B_{2 n}\right|(2 n-1) x^{2 n-2}, \\
& \frac{1}{(\sinh x)^{2}}=-(\operatorname{coth} x)^{\prime}=\frac{1}{x^{2}}-\sum_{n=1}^{\infty} \frac{2^{2 n}}{(2 n) !}(2 n-1)\left|B_{2 n}\right| x^{2 n-2} .
\end{aligned}
$$

For the following expansion formula

$$
\frac{x}{\sin x}=1+\sum_{n=1}^{\infty} \frac{2^{2 n}-2}{(2 n) !}\left|B_{2 n}\right| x^{2 n}
$$

see [8].

Lemma 2 (Theorem 2 in [1]). For $-\infty<a<b<\infty$, let $f, g:[a, b] \rightarrow \mathbb{R}$ be continuous on $[a, b]$, and differentiable on $(a, b)$. Let $g^{\prime}(x) \neq 0$ on $(a, b)$. If $f^{\prime}(x) / g^{\prime}(x)$ is increasing (decreasing) on ( $a, b)$, then so are

$$
\frac{f(x)-f(a)}{g(x)-g(a)} \text { and } \frac{f(x)-f(b)}{g(x)-g(b)} .
$$

If $f^{\prime}(x) / g^{\prime}(x)$ is strictly monotone, then the monotonicity in the conclusion is also strict.

Lemma 3. The following function

$$
f_{1}(x)=\frac{(x / \sin x)^{2}-x \cot x}{1-x \cot x}
$$

is strictly increasing from $(0, \pi / 2)$ onto $\left(\pi^{2} / 4\right)$.

Proof. Let $f_{1}(x)=A_{1}(x) / C_{1}(x)$, where

$$
A_{1}(x)=(x / \sin x)^{2}-x \cot x \text { and } C_{1}(x)=1-x \cot x .
$$

By using expansion formulas (2.2) and (2.4) we get

$$
A_{1}(x)=1+\sum_{n=1}^{\infty} \frac{2^{2 n}}{(2 n) !}(2 n-1)\left|B_{2 n}\right| x^{2 n}-1+\sum_{n=1}^{\infty} \frac{2^{2 n}}{(2 n) !}\left|B_{2 n}\right| x^{2 n}
$$




$$
=\sum_{n=1}^{\infty} \frac{2^{2 n} 2 n}{(2 n) !}\left|B_{2 n}\right| x^{2 n}=\sum_{n=1}^{\infty} a_{n} x^{2 n}
$$

and

$$
C_{1}(x)=\sum_{n=1}^{\infty} \frac{2^{2 n}}{(2 n) !}\left|B_{2 n}\right| x^{2 n}=\sum_{n=1}^{\infty} c_{n} x^{2 n} .
$$

Let $d_{n}=a_{n} / c_{n}=2 n$, which is increasing in $n \in \mathbb{N}$. Thus, by Lemma $1 f_{1}(x)$ is strictly increasing in $x \in(0, \pi / 2)$. Applying l'Hôspital rule, we get $\lim _{x \rightarrow 0} f_{1}(x)=$ 2 and $\lim _{x \rightarrow \pi / 2} f_{1}(x)=\pi^{2} / 4$. This completes the proof.

Lemma 4. The following inequalities hold

$$
\begin{aligned}
\frac{\tanh x}{x} & \leq \frac{2}{\sqrt{9+4 x^{2}}-1}, \quad x \in \mathbb{R}, \\
\frac{\sinh x}{x}<\frac{\cosh x+2}{3} & <(\cosh x)^{1 / 3} \frac{\cosh (2 x / 3)+1}{2}, \quad x>0 .
\end{aligned}
$$

Proof. Clearly both sides of the inequality (2.7) get value 1 at $x=0$. By symmetry of the function we need to consider only the positive values of $x$. Let

$$
f_{2}(x)=\left(\frac{2 x}{\tanh x}+1\right)^{2}-4 x^{2}-9 .
$$

By differentiation we get

$$
\begin{aligned}
f_{2}^{\prime}(x) & =4\left(\operatorname{coth} x+\frac{x(1-2 x \operatorname{coth} x)}{(\sinh x)^{2}}\right) \\
& =4 \frac{x^{2} \cosh x}{(\sinh x)^{3}}\left(\left(\frac{\sinh x}{x}\right)^{2}+\frac{\tanh x}{x}-2\right) \\
& =4 \frac{x^{2} \cosh x}{(\sinh x)^{3}} f_{3}(x)>0,
\end{aligned}
$$

where the last inequality follows since $f_{3}(x)>0$ is equivalent to

$$
\frac{\sinh x}{x}+\frac{1}{\cosh x}>\frac{x}{\sinh x}
$$

which is clearly true because $(\sinh x) / x>1$. Now $f_{2}$ is strictly increasing, and $\lim _{x \rightarrow 0} f_{2}(x)=0<f_{2}(x)$. This implies the proof of (2.7).

The first inequality in (2.8) is well known, for the second inequality we define

$$
f_{4}(x)=\frac{\cosh x+2}{3}-(\cosh x)^{1 / 3} \frac{\cosh (2 x / 3)+1}{2} .
$$

Simple computation gives

$$
f_{4}^{\prime}(x)=\frac{\sinh \frac{x}{3}-2 \sinh x+4 \cosh ^{2 / 3} x \sinh x-3 \sinh \frac{5 x}{3}}{12(\cosh x)^{2 / 3}}
$$


and clearly $\sinh \frac{x}{3}-2 \sinh x<0$. We prove that $4 \cosh ^{2 / 3} x \sinh x-3 \sinh \frac{5 x}{3}<0$, which is equivalent to

$$
f_{5}(x)=4 \sinh x+2 \sinh (3 x)+\frac{11}{8} \sinh (5 x)-\frac{81}{8} \sinh \frac{5 x}{3}>0 .
$$

Since

$$
\begin{aligned}
f_{5}^{\prime \prime}(x) & =4 \sinh x+18 \sinh (3 x)+\frac{275}{8} \sinh (5 x)-\frac{225}{8} \sinh \frac{5 x}{3} \\
& \geq \frac{275}{8} \sinh (5 x)-\frac{225}{8} \sinh \frac{5 x}{3}>0
\end{aligned}
$$

for all $x>0$, it is clear that $f_{5}^{\prime}(x)>f_{5}^{\prime}(0)=0$ and $f_{5}(x)$ is increasing. Thus $f_{5}(x)>$ $f_{5}(0)=0$.

Remark 4. In Lemma (2.7) the difference between the function and the upper bound is less than 0.02 . The upper bound is asymptotically sharp $\frac{2}{\sqrt{9+4 x^{2}-1}}-$ $\frac{\tanh x}{x} \rightarrow 0$ as $x \rightarrow \pm \infty$.

\section{Proofs of the MAin Results}

In this section we prove our main theorems.

Proof of Theorem 1. Sharpness of the bounds is obvious. Since the bounds and the function $\frac{\sin x}{x}$ are even we need to prove sharp inequality for $x \in(0, \pi / 2)$. Let

$$
f_{6}(x)=\frac{\cos x-1}{(\sin x) / x-1}=\frac{(\sin x) / x-x \cot x}{x / \sin x-1}=\frac{A_{2}(x)}{C_{2}(x)} .
$$

Using series expansion formulas (2.1) and (2.6), we get

$$
\begin{aligned}
A_{2}(x) & =\sum_{n=1}^{\infty} \frac{2^{2 n}-2}{(2 n) !}\left|B_{2 n}\right| x^{2 n}+\sum_{n=1}^{\infty} \frac{2^{2 n}}{(2 n) !}\left|B_{2 n}\right| x^{2 n} \\
& =\sum_{n=1}^{\infty} \frac{2\left(2^{2 n}-1\right)}{(2 n) !}\left|B_{2 n}\right| x^{2 n}=\sum_{n=1}^{\infty} \tilde{a}_{n} x^{2 n},
\end{aligned}
$$

and

$$
C_{2}(x)=\sum_{n=1}^{\infty} \frac{2^{2 n}-2}{(2 n) !}\left|B_{2 n}\right| x^{2 n}=\sum_{n=1}^{\infty} \tilde{c}_{n} x^{2 n} .
$$

We denote $\tilde{d}=\tilde{a} / \tilde{c}=2\left(2^{2 n}-1\right) /\left(2^{2 n}-2\right)$. Clearly $\tilde{d}$ is a decreasing function of $n \in \mathbb{N}$. Hence $f_{6}$ is decreasing by Lemma 1. Applying l'Hôspital rule, we get $\lim _{x \rightarrow 0} f_{6}(x)=3$ and $\lim _{x \rightarrow \pi / 2} f_{6}(x)=\pi /(\pi-2)$, this finishes the proof. 
Proof of Theorem 2. Equality in the claim is clearly attained at points $x=-\pi / 2$, $x=0$ and $x=\pi / 2$. Since the left hand side of the inequality is an even function we need to show that the inequality is sharp for $x \in(0, \pi / 2)$. Let

$$
\frac{x / \sin x-\cos x}{(\sin x) / x-x \cot x}=\frac{(x / \sin x)^{2}-x \cot x}{1-x \cot x}=f_{1}(x) .
$$

By Lemma 3, we get

$$
2<\frac{x / \sin x-\cos x}{(\sin x) / x-x \cot x}<\frac{\pi^{2}}{4},
$$

which implies the following inequalities

$$
\frac{4}{\pi^{2}}\left(\frac{x}{\sin x}+\left(\frac{\pi^{2}}{4}-1\right) \cos x\right)<\frac{\sin x}{x}<\frac{1}{2}\left(\frac{x}{\sin x}+\cos x\right) .
$$

The first inequality of (3.1) can be written as (1.7). This completes the proof.

The second inequality of (3.1) is also proved by Neuman and Sándor, see [11, Theorem 2.3]. They pointed out that it can be written as the Wu-Srivastava inequality.

Lemma 5. Let $\alpha=\pi /(\pi-2)$ as in Theorem 1. The function

$$
f_{\alpha}(b)=\left(\frac{\alpha}{\alpha+b-1}\right)^{\alpha}+\frac{\alpha b}{\alpha+b-1}
$$

is decreasing from $(0,1)$ onto $(2, k)$, where $k=(\pi / 2)^{\alpha} \approx 3.46505$.

Proof. By differentiation we obtain

$$
\begin{aligned}
f_{\alpha}^{\prime}(b) & =\frac{a\left(a-1-(a+b-1)(a /(a+b-1))^{a}\right)}{(a+b-1)^{2}} \\
& =-\frac{\pi\left(\pi^{\pi /(\pi-2)}(2+\pi(\pi-2))^{(2-\pi) / 2}-2\right)}{(2+b(\pi-2))^{2}},
\end{aligned}
$$

which is negative, and $f_{\alpha}(b) \rightarrow k$ as $b \rightarrow 0$ and $f_{\alpha}(b) \rightarrow 2$ as $b \rightarrow 1$.

Proof of Theorem 3. The inequality (1.8) follows from the first inequality of (1.6). Again, utilizing the same inequality

$$
\frac{x}{\sin x}<\frac{\alpha}{\alpha-1+\cos x}
$$

we get

$$
\left(\frac{x}{\sin x}\right)^{\alpha}+\frac{x}{\tan x}<\left(\frac{\alpha}{\alpha-1+\cos x}\right)^{\alpha}+\frac{\alpha \cos x}{\alpha-1+\cos x}=f_{\alpha}(\cos x)<\left(\frac{\pi}{2}\right)^{\alpha}
$$

by Lemma 5 , because $f_{\alpha}(\cos x)$ is strictly increasing and $\lim _{x \rightarrow 1} f_{\alpha}(\cos x)=(\pi / 2)^{\alpha}$. 
An other proof for (1.8). Let

$$
f(x)=(\alpha-1) \frac{x}{\sin x}+\frac{x}{\tan x}
$$

for $x \in(0, \pi / 2)$. An easy computation gives

$$
(\sin x)^{2} \cdot f^{\prime}(x)=(\alpha-1) \sin x-(\alpha-1) x \cos x+\sin x \cos x-x=g(x) .
$$

Now $g^{\prime}(x)=(2(\sin x) / x) \cdot h(x)$, where $h(x)=(\alpha-1) / 2-(\sin x) / x$. The function $(\sin x) / x$ is strictly decreasing as the function of $x$, and thus the equation $(\alpha-1) / 2=$ $(\sin x) / x$ has at most a single root in $(0, \pi / 2)$.

Suppose that $(\alpha-1) / 2<1$, then the equation has exactly one root $x_{0} \approx 0.8795$. As $h(x)<0$ for $x<x_{0}$ and $h(x)>0$ for $x>x_{0}$, the function $g(x)$ will be strictly decreasing for $x$ in $\left(0, x_{0}\right)$, and strictly increasing in $\left(x_{0}, \pi / 2\right)$.

Suppose that $\alpha>\pi / 2+1$, which is equivalent to $(\alpha-1) / 2>\pi / 4$. Then we get $g(\pi / 2)=\alpha-1-\pi / 2>0$. As $g(0)=0$ we have $g\left(x_{0}\right)<0$, and $g$ will have a single root $x_{1}$ in interval $\left(x_{0}, \pi / 2\right)$. Then we get $g(x)<0$ for $x$ in $\left(0, x_{1}\right)$, and $g(x)>0$ for $x$ in $\left(x_{1}, \pi / 2\right)$, where $x_{1} \approx 1.1559$. This means that at the points 0 and $\pi / 2$ the function $f(x)$ will obtain its maximum values. If $f(\pi / 2) \leq f(0)$, the inequality $f(x) \leq \alpha$ is true. The inequality $f(\pi / 2) \leq f(0)$ is equivalent to $\alpha \leq \pi /(\pi-2)$. Remark that for the best possible $\alpha=\pi /(\pi-2)$ one has also $\alpha>\pi / 2+1$ and $(\alpha-1) / 2<1$, so the assumed properties in the proof are valid. This finishes the proof of the inequality $f(x) \leq \alpha$, with best possible $\alpha=\pi /(\pi-2)$.

Remark 5. Indeed, the point $x_{1}$ above is the minimum point of $f(x)$ on $(0, \pi / 2)$. In fact the following converse of inequality (1.8)

$$
(\alpha-1) \frac{x}{\sin x}+\frac{x}{\tan x} \geq f\left(x_{1}\right) \approx 2.7219
$$

holds true for $\alpha=\pi /(\pi-2),(\alpha-1) / 2<1$ and $\alpha>\pi / 2+1$ (as $\alpha<3$, it is sufficient to suppose $\pi / 2+1<\alpha \leq \pi /(\pi-2))$.

Proof of Theorem 4. Clearly the function, its lower and upper bound get value 3 at origin. By symmetry of the function we consider only values $x \in(0, \pi / 2)$. The second inequality in (1.10) is equivalent to write

$$
f_{7}(x)=\left(\frac{2+\cos x}{3 \cos x}\right)-\left(\frac{2 x}{\sin x}+\frac{x}{\sin x \cos x}\right)>0 .
$$

It is sufficient to prove that $f_{7}>0$. Using the the following inequalities

we get

$$
(\cos (x / 2))^{4 / 3}<\frac{\sin x}{x}<\frac{2+\cos x}{3}
$$

$$
f_{7}(x)>\frac{3}{\cos x} \frac{\sin x}{x}-\frac{x}{\sin x}\left(2+\frac{1}{\cos x}\right)
$$




$$
\begin{aligned}
& =\frac{3 x}{\sin x \cos x}\left(\left(\frac{\sin x}{x}\right)^{2}-\frac{2 \cos x+1}{3}\right) \\
& >\frac{3 x}{\sin x \cos x}\left(\left(\frac{1+\cos x}{2}\right)^{4 / 3}-\frac{2 \cos x+1}{3}\right) \\
& =\frac{6 x}{\sin 2 x} f_{8}(x) .
\end{aligned}
$$

For showing that $f_{8}$ is positive, we define the function

$$
f_{9}(y)=\frac{(1+y)^{4}}{(2 y+1)^{3}}, \quad y \in(0,1),
$$

and get

$$
f_{9}^{\prime}(y)=\frac{(1-y)(1+y)^{3}}{(1+2 y)^{4}}<0,
$$

with $f_{9}(1)=16 / 27$. This implies that $f_{8}$ is positive, and this completes the proof of the second inequality.

Remark 6. The upper bound of Theorem 4 holds true for values $x \in(-\pi, \pi)$. The difference between the function and the lower bound is less than 1.6 and between the function and the upper bound is less than 0.55 . In both cases the difference is less than $x^{2}$.

Corollary 1. For $x \in(0, \pi / 2)$, we have

$$
\frac{\pi}{2}+\cos x<\frac{x}{\sin x}+2 \frac{x}{\tan x}<2+\cos x .
$$

Proof. Let $f(x)=x / \sin x+2 x / \tan x-\cos x$ for $x \in(0, \pi / 2)$. After elementary computations, we get

$$
f^{\prime}(x) \cdot(\sin x)^{2}=\sin x-x \cos x+2 \sin x \cos x-2 x+(\sin x)^{3}=h(x) .
$$

Now $h^{\prime}(x)=\sin x \cdot k(x)$, where

$$
k(x)=x-4 \sin x+3 \sin x \cos x .
$$

As $k^{\prime}(x)=4(\cos x)^{2}-4 \cos x-2(\sin x)^{2}$, and $0<\cos x<1$ we obtain $k^{\prime}(x)<$ 0 . Thus $k(x)<k(0)=0$, implying $h(x)<h(0)=0$. Finally, we get $f^{\prime}(x)<0$. Thus $f(x)$ is strictly decreasing, this implies $f(\pi / 2)<f(x)<f(0)$, so the result follows.

We remark here that the first inequality of (3.3) cannot be compared with the first inequality of (1.10), and obviously the right hand sides of both inequalities are equal.

Corollary 2. The inequality (2.7) implies the hyperbolic version of the WuSrivastava inequality (1.5). 
Proof. It is easy to see that the inequality (2.7) is equivalent to

$$
\left(\frac{x}{\tanh x}\right)^{2}+\frac{x}{\tanh x}-x^{2}-2>0 .
$$

The above inequality can be written as

$$
\left(\frac{x}{\sinh x}\right)^{2}+\frac{x}{\tanh x}>0
$$

by observing that

$$
\left(\frac{x}{\sinh x}\right)^{2}-x^{2}=\frac{x}{\sinh x}>0 .
$$

Proof of Theorem 5. The first inequality is well known and follows from (1.2). Similarly the second inequality follows from (2.8).

In $2010 \mathrm{~J}$. Sándor (see [15]) proved that the best positive constants $a$ and $b$ such that

$$
\left(\frac{\cos x+2}{3}\right)^{a}<\frac{\sin x}{x}<\left(\frac{\cos x+2}{3}\right)^{b},
$$

are $a=\log (\pi / 2) / \log (3 / 2) \approx 1.113$ and $b=1$.

Now, we state two similar results:

Theorem 7. For $x \in(-\pi / 2, \pi / 2)$, we have

(1) $\frac{\cos x+2}{3^{\alpha_{1}}}<\frac{\sin x}{x}<\frac{\cos x+2}{3^{\beta_{1}}}$,

(2) $\frac{\cos x+2^{\alpha_{2}}}{3}<\frac{\sin x}{x}<\frac{\cos x+2^{\beta_{2}}}{3}$,

with best possible constants $\alpha_{1}=\log (\pi) / \log (3) \approx 1.04198, \alpha_{2}=\log (\pi / 6) / \log (2) \approx$ $0.93345, \beta_{1}=1$ and $\beta_{2}=1$.

Proof. For (1), let $f_{10}(x)=\frac{x(2+\cos x)}{\sin x}$, with $x \in(0, \pi / 2)$. One has

$$
(\sin x)^{2} \cdot f_{10}^{\prime}(x)=2 \sin x-2 x \cos x+\sin x \cos x-x=f_{11}(x) .
$$

As $f_{11}^{\prime}(x)=2(\sin x)(x-\sin x)>0$, we get $f_{11}(x)>f_{11}(0)=0$, so $f_{10}^{\prime}(x)>0$, proving that $f_{10}(x)$ is strictly increasing. This implies that the function

$$
f_{12}(x)=\frac{1}{\log 3} \log \left(\frac{x(\cos x+2)}{\sin x}\right)
$$

is strictly increasing in $x \in(0, \pi / 2)$, and we get

$$
\beta_{1}=f_{12}(0+)<f_{12}(x)<f_{12}(\pi / 2)=\alpha_{1},
$$

thus (1) follows.

To prove (2) we write $f_{13}=3(\sin x) / x-\cos x$, for $x \in(0, \pi / 2)$. Now

$$
x^{2} f_{13}^{\prime}(x)=3 x \cos x+x^{2} \sin x-3 \sin x=f_{14}(x),
$$


and $f_{14}^{\prime}(x)=x(x \cos x-\sin x)<0$, since $x \cos x<\sin x$. This shows that $f_{14}(x)<$ $f_{14}(0)=0$, implying $f_{13}^{\prime}(x)<0$ and proving that $f_{13}(x)$ is strictly decreasing. Now $f_{13}(x)<f_{13}(0+)=2$ and $f_{13}(x)>f_{13}(\pi / 2)=6 / \pi$. As $2=2^{\beta_{2}}$ for $\beta_{2}=1$, and $6 / \pi=2^{\alpha_{2}}$ for $\left.\alpha_{2}=\log (6 / \pi)\right) / \log (2)$, the result follows.

Proof of Theorem 6. Let

$$
f(x)=\log \left(\frac{(\pi-2) \cos x+2}{\pi}\right)+\frac{(\pi-2) x^{2}}{2 \pi} .
$$

Simple calculation yields

$$
f^{\prime}(x)=(\pi-2)\left(\frac{x}{\pi}-\frac{\sin x}{2+(\pi-2) \cos x}\right),
$$

which is negative by Theorem 1 . Thus, the $f$ function is strictly decreasing, and

$$
\alpha=\lim _{x \rightarrow \pi / 2} f(x)<f(x)<0=\beta=\lim _{x \rightarrow 0} f(x) .
$$

This implies the proof.

We finish the paper by giving a new type of Kober's inequality [9, 14], which follows easily from (1.11) and Theorem 6.

Corollary 3. For $x \in(0, \pi / 2)$, the following inequalities hold

$$
3 \exp \left(-\frac{x^{2}}{6}\right)-2<\cos x<\frac{\pi \exp \left(-(\pi-2) x^{2} /(2 \pi)\right)-2}{\pi-2} .
$$

\section{REFERENCES}

[1] G. Anderson, M. Vamanamurthy, and M. Vuorinen, "Monotonicity rules in calculus," Amer. Math. Monthly, vol. 113, no. 9, pp. 805-816, 2006, doi: 10.2307/27642062. [Online]. Available: http://dx.doi.org/10.2307/27642062

[2] B. A. Bhayo and J. Sándor, “On Jordan's, Redheffer's and Wilker's inequality,” Math. Inequal. Appl., vol. 19, no. 3, pp. 823-839, 2016, doi: 10.7153/mia-19-60. [Online]. Available: http://dx.doi.org/10.7153/mia-19-60

[3] B.-N. Guo, B.-M. Qiao, F. Qi, and W. Li, "On new proofs of Wilker's inequalities involving trigonometric functions," Math. Inequal. Appl., vol. 6, no. 1, pp. 19-22, 2003, doi: 10.7153/mia-06-02. [Online]. Available: http://dx.doi.org/10.7153/mia-06-02

[4] V. Heikkala, M. K. Vamanamurthy, and M. Vuorinen, "Generalized elliptic integrals," Comput. Methods Funct. Theory, vol. 9, no. 1, pp. 75-109, 2009, doi: 10.1007/BF03321716. [Online]. Available: http://dx.doi.org/10.1007/BF03321716

[5] C. Huygens, "Oeuvres completes," Société Hollondaise des Science, 1888.

[6] K. Ireland and M. Rosen, A classical introduction to modern number theory, 2nd ed., ser. Graduate Texts in Mathematics. Springer-Verlag, New York, 1990, vol. 84. [Online]. Available: http://dx.doi.org/10.1007/978-1-4757-2103-4. doi: 10.1007/978-1-4757-2103-4

[7] A. Jeffrey, Handbook of mathematical formulas and integrals, 3rd ed. Elsevier Academic Press, San Diego, CA, 2004. 
[8] J.-L. Li, “An identity related to Jordan's inequality," Int. J. Math. Math. Sci., pp. Art. ID 76782, 6, 2006, doi: 10.1155/IJMMS/2006/76782. [Online]. Available: http: //dx.doi.org/10.1155/IJMMS/2006/76782

[9] D. S. Mitrinović, Analytic inequalities. Springer-Verlag, New York-Berlin, 1970, in cooperation with P. M. Vasić. Die Grundlehren der mathematischen Wissenschaften, Band 165.

[10] H. L. Montgomery, J. D. Vaaler, J. Delany, D. E. Knuth, D. Vialetto, M. S. Klamkin, and J. B. Wilker, "Problems and Solutions: Elementary Problems: E3301-E3306," Amer. Math. Monthly, vol. 96, no. 1, pp. 54-55, 1989, doi: 10.2307/2323259. [Online]. Available: http://dx.doi.org/10.2307/2323259

[11] E. Neuman and J. Sándor, "On some inequalities involving trigonometric and hyperbolic functions with emphasis on the Cusa-Huygens, Wilker, and Huygens inequalities," Math. Inequal. Appl., vol. 13, no. 4, pp. 715-723, 2010, doi: 10.7153/mia-13-50. [Online]. Available: http://dx.doi.org/10.7153/mia-13-50

[12] I. Pinelis, "l'Hospital rules for monotonicity and the Wilker-Anglesio inequality," Amer. Math. Monthly, vol. 111, no. 10, pp. 905-909, 2004, doi: 10.2307/4145099. [Online]. Available: http://dx.doi.org/10.2307/4145099

[13] Q. I. Rahman, "On the monotonicity of certain functionals in the theory of analytic functions," Canad. Math. Bull., vol. 10, pp. 723-729, 1967.

[14] J. Sándor, "On new refinements of Kober's and Jordan's inequality," Notes on number theory and discrete mathematics, vol. 19, no. 1, pp. 73-83, 2013.

[15] J. Sándor, "Sharp Cusa-Huygens and related inequalities," Notes on number theory and discrete mathematics, vol. 19, no. 1, pp. 50-54, 2013.

[16] J. Sándor and M. Bencze, "On Huygens' trigonometric inequality," RGMIA Res. Rep. Collection, vol. 8, no. 3, 2005

[17] J. B. Wilker, J. S. Sumner, A. A. Jagers, M. Vowe, and J. Anglesio, "Problems and Solutions: Solutions of Elementary Problems: E3306," Amer. Math. Monthly, vol. 98, no. 3, pp. 264-267, 1991, doi: 10.2307/2325035. [Online]. Available: http://dx.doi.org/10.2307/2325035

[18] S.-H. Wu and H. M. Srivastava, "A weighted and exponential generalization of Wilker's inequality and its applications," Integral Transforms Spec. Funct., vol. 18, no. $7-$ 8, pp. 529-535, 2007, doi: 10.1080/10652460701284164. [Online]. Available: http: //dx.doi.org/10.1080/10652460701284164

[19] S.-H. Wu and H. M. Srivastava, "A further refinement of Wilker's inequality," Integral Transforms Spec. Funct., vol. 19, no. 9-10, pp. 757-765, 2008, doi: 10.1080/10652460802340931. [Online]. Available: http://dx.doi.org/10.1080/10652460802340931

[20] Z.-H. Yang, "Refinements of a two-sided inequality for trigonometric functions," J. Math. Inequal., vol. 7, no. 4, pp. 601-615, 2013, doi: 10.7153/jmi-07-57. [Online]. Available: http://dx.doi.org/10.7153/jmi-07-57

[21] L. Zhu, "A new simple proof of Wilker's inequality," Math. Inequal. Appl., vol. 8, no. 4, pp. 749750, 2005, doi: 10.7153/mia-08-70. [Online]. Available: http://dx.doi.org/10.7153/mia-08-70

[22] L. Zhu, "On Wilker-type inequalities," Math. Inequal. Appl., vol. 10, no. 4, pp. 727-731, 2007, doi: 10.7153/mia-10-67. [Online]. Available: http://dx.doi.org/10.7153/mia-10-67

Authors' addresses

Barkat Ali Bhayo

Department of Mathematics \& Statistics, Quaid-e-Awam University of Engineering Science \& Technology Nawabshah, Pakistan

E-mail address: bhayo.barkategmail.com 
Riku Klén

Department of Mathematics and Statistics, University of Turku, FI-20014 Finland

E-mail address: riku.klen@utu.fi

József Sándor

Babeş-Bolyai University, Department of Mathematics, Str. Kogalniceanu nr. 1, 400084 ClujNapoca, Romania

E-mail address: jsandor@math.ubbcluj.ro 